\title{
Efeito do ácido zoledrônico e do alendronato no edema ósseo e dor na osteonecrose espontânea do joelho: Um novo paradigma no manejo*
}

\section{Effect of Zoledronic Acid and Alendronate on Bone Edema and Pain in Spontaneous Osteonecrosis of the Knee: A New Paradigm in the Medical Management}

\author{
Sanjay Agarwala ${ }^{11} \quad$ Lokesh Sharoff $^{1} \quad$ Naeem Jagani ${ }^{1}$ \\ ${ }^{1}$ Hinduja Hospital and Medical Research Centre, Mumbai, Índia \\ Rev Bras Ortop 2020;55(5):543-550. \\ Endereço para correspondência Sanjay Agarwala, MS, Hinduja \\ Hospital and Medical Research Centre, Mumbai, 400016, Índia \\ (e-mail: drsa2011@gmail.com).
}

\section{Resumo}

Palavras-chave

- necrose

- bisfosfonatos

- osteoartrite

- articulação do joelho

Abstract
Objetivo Determinar o efeito do tratamento combinado de ácido zoledrônico e alendronato no edema ósseo e na dor no joelho em casos de osteonecrose espontânea do joelho. A experiência dos autores com este tratamento é relatada.

Métodos Série de casos retrospectiva, incluindo 11 pacientes com osteonecrose espontânea do joelho confirmada por ressonância magnética. Os pacientes foram tratados com uma dose intravenosa única de $5 \mathrm{mg}$ de ácido zoledrônico combinada com $35 \mathrm{mg}$ de alendronato oral, 2 vezes por semana, por 16 semanas. Os escores da escala visual analógica foram aferidos antes do começo do tratamento, em 8 semanas e em 16 semanas de acompanhamento. O tamanho do edema da medula óssea adjacente à lesão foi medido em imagens de ressonância magnética coronal ponderadas em $\mathrm{T} 2$ no início do tratamento e em 16 semanas.

Resultados 0 escore médio da escala visual analógica em 0 semanas foi de 7,72, contra 0,81 em 16 semanas de tratamento, uma diferença estatisticamente significativa $(p=0,03)$. O envolvimento médio da medula óssea em 0 semanas foi de $80 \%$, e foi reduzido para $11,81 \%$ em 16 semanas de tratamento, uma diferença também estatisticamente significativa $(p=0,03)$.

Conclusão Os dados mostram que a terapia combinada proporciona alívio da dor inicial e redução do edema ósseo, sendo segura, eficaz e bem tolerada em uma enfermidade dolorosa como a osteonecrose espontânea do joelho.

Objective The aim of the present study was to determine the effect of combined zoledronic acid and alendronate therapy on bone edema and knee pain in cases of spontaneous osteonecrosis of the knee. We report our experience with this treatment.
Estudo realizado no Hinduja Hospital and Medical Research Centre, Mumbai, Índia. Publicado Originalmente por Elsevier Editora Ltda. recebido

30 de Julho de 2017

aceito

07 de Dezembro de 2017
DOI https://doi.org/

10.1016/j.rboe.2017.12.008. ISSN 0102-3616.
Copyright $\odot 2020$ by Sociedade Brasileira License terms de Ortopedia e Traumatologia. Published by Thieme Revnter Publicações Ltda, Rio de Janeiro, Brazil 


\section{Keywords}

- necrosis

- bisphosphonates

- osteoarthritis

- knee joint
Methods A retrospective case series of 11 patients with spontaneous osteonecrosis of the knee confirmed by magnetic resonance image (MRI). The patients were treated with a single dose of $5 \mathrm{mg}$ of intravenous zoledronic acid combined with $35 \mathrm{mg}$ twice a week of oral alendronate, for 16 weeks. The visual analogue scale scores were noted before the beginning of the therapy, at 8 weeks, and at 16 weeks of follow-up. The size of the bone marrow edema adjacent to the lesion was measured on T2-weighted MRI coronal images at the beginning of the therapy and at 16 weeks.

Results The average visual analogue scale score at 0 weeks was of 7.72 , and of 0.81 at 16 weeks of therapy; the difference was statistically significant $(p=0.03)$. The mean bone marrow involvement at 0 weeks was of $80 \%$, which reduced to $11.81 \%$ at 16 weeks of therapy. This change was statistically significant $(p=0.03)$.

Conclusion Our data shows that the combination therapy causes early pain relief and reduction of the bone edema, and it is safe, effective and well-tolerated for a painful disease entity like spontaneous osteonecrosis of the knee.

\section{Introdução}

A osteonecrose espontânea do joelho (ONEJ) foi inicialmente descrita por Ahlbäck et al. ${ }^{1}$ em 1968 . A osteonecrose, que originalmente fora descrita como uma entidade única, foi então reclassificada em três quadros diferentes: 1) ONEJ, que normalmente afeta um único côndilo em pacientes mais velhos; 2) osteonecrose secundária, quando o quadro tem uma causa conhecida, tal como anemia falciforme, tratamento com corticosteroides etc., na qual pacientes mais jovens são mais comumente afetados, e o aspecto radiográfico é distinto do de lesões que envolvem focos múltiplos; e 3 ) osteonecrose pós-artroscópica do joelho, que afeta apenas um côndilo. 0 início dos sintomas é aumento da dor no joelho com resultados positivos de ressonância magnética (RM). ${ }^{2,3}$ Yamamoto et al. ${ }^{4}$ concluíram, com base em suas descobertas em amostras histológicas de 14 pacientes no pós-operatório, que a fratura de insuficiência subcondral que resulta da osteoporose subjacente é a etiologia da ONEJ. Geralmente, os pacientes de ONEJ têm 55 anos ou mais, não apresentam fatores de risco, e apresentam dor monoarticular unilateral..$^{5}$ As mulheres são mais comumente afetadas. ${ }^{6}$ As duas principais teorias etiológicas são trauma e doenças vasculares. ${ }^{7,8}$ As investigações incluem radiografia anteroposterior, lateral e oblíqua da articulação do joelho, embora a RM seja necessária para fazer o diagnóstico. No curso inicial da doença, as radiografias são frequentemente negativas e, em alguns casos, podem permanecer negativas pela duração total dos sintomas clínicos. ${ }^{9} \mathrm{Tem}$ sido presumido que a suscetibilidade do côndilo femoral medial à ONEJ seja resultado de um suprimento de sangue variável entre os côndilos medial e lateral. Um estudo com cadáveres realizado por Reddy e Fredericks ${ }^{11}$ demonstrou que o côndilo femoral medial tem suprimento sanguíneo intraósseo limitado, com áreas irrigadas definidas e aparentes, enquanto o côndilo femoral lateral tem um rico suprimento vascular tanto extra quanto intraósseo. ${ }^{10,11}$ Satku et al. ${ }^{12}$ também descreveram a ONEJ do platô tibial medial proximal, que é progressiva na maioria dos casos, levando à doença articular degenerativa. Lotke et al. ${ }^{13}$ descreveram que os sintomas de pacientes não tratados que têm evidências radiológicas de ONEJ podem durar até sete anos após o diagnóstico desta condição dolorosa. $\mathrm{O}$ tratamento de ONEJ é tradicionalmente feito tanto de forma cirúrgica quanto não cirurgicamente. O manejo não cirúrgico da ONEJ inclui suporte de peso protegido, medicamentos anti-inflamatórios não esteroides (AINES), bisfosfonatos e prostaglandinas. O manejo cirúrgico inclui descompressão central por perfuração, enxerto ósseo, enxerto osteocondral, artroscopia, osteotomia tibial, e artroplastia total ou unicompartimental do joelho. ${ }^{10} \mathrm{O}$ tratamento atual de ONEJ parece insatisfatório. A patologia da ONEJ é semelhante à da necrose avascular da cabeça femoral. Agarwala et al. ${ }^{14}$ relataram 16 pacientes em seu estudo piloto com necrose avascular do quadril com o uso de alendronato. Esse estudo foi então seguido por 8 anos em 395 quadris com necrose avascular (NAV). ${ }^{15}$ Com essa terapia, foi verificada melhora significativa na dor já com 12 semanas, e melhoria da capacidade funcional com a redução da necessidade de analgésicos. Pelo que sabemos, existem apenas quatro artigos que mencionam os resultados do uso de bisfosfonatos especificamente para as lesões de ONEJ. ${ }^{3,16-18}$

\section{Materiais e Métodos}

Os dados de 11 pacientes com lesões de ONEJ envolvendo apenas o fêmur foram analisados entre março de 2015 e julho de 2016. Este estudo foi devidamente aprovado pelo conselho de revisão institucional. Todos os pacientes incluídos tiveram um diagnóstico comprovado de ONEJ por RM. Os critérios de exclusão foram: causa secundária conhecida de osteonecrose; distúrbio renal; histórico de traumatismo; distúrbios ósseos que interferissem nos resultados da RM; alergia a bisfosfonatos (casos em que o ácido zoledrônico [AZ] ou o alendronato não podem ser administrados); e acompanhamento de menos de 16 semanas). A medicação foi iniciada imediatamente: uma injeção de dose única de $5 \mathrm{mg}$ de $\mathrm{AZ}$ foi administrada, assim como comprimidos de $70 \mathrm{mg}$ de alendronato semanalmente em 2 doses divididas e ingeridas com o estômago vazio. Isso foi suplementado com cálcio, vitamina D e 
medicamentos anti-inflamatórios. Essa terapia permitiu a possibilidade de interromper a terapêutica oral com alendronato em caso de efeitos secundários indesejáveis. Nos três meses iniciais de tratamento, todos os pacientes foram aconselhados a utilizar suporte parcial de peso com um andador, e, mais tarde, o suporte de peso foi aumentado gradualmente, conforme permitido pela dor. $\mathrm{O}$ escore da escala visual analógica (EVA) foi anotado antes de se iniciar a terapia, com 8 semanas e depois com 16 semanas de acompanhamento (em uma escala de 0 a 10 , com 0 indicando ausência de dor, e 10 indicando a pior dor possível). A RM e as radiografias da articulação do joelho afetado foram repetidas com 16 semanas de tratamento. $O$ tamanho do edema da medula óssea (EMO) foi medido em imagens coronais de RM ponderadas em T2, calculando-se a largura máxima da lesão que afeta a superfície articular na visão coronal como uma porcentagem do côndilo femoral afetado. Isso foi medido no início da terapia e no acompanhamento de 16 semanas. Exames de RM não foram realizados antes de 16 semanas. 0 edema da medula óssea foi avaliado por áreas de diminuição da intensidade do sinal do osso subcondral na RM ponderada por densidade protônica (DP) e aumento da intensidade do sinal nas imagens ponderadas em T2. A RM foi interpretada por um radiologista experiente que não fazia parte do estudo.

\section{Análise Estatística}

Os dados foram registrados em planilhas do programa Excel (Microsoft, Redmond, WA, EUA). As características demográficas básicas foram registradas. Com base na normalidade, a comparação do escore da EVA entre a pré e a pós-terapia e a comparação do EMO foram feitas utilizando-se o teste dos postos sinalizados de Wilcoxon. Valores de $p \leq 0.05$ foram considerados estatisticamente significativos.

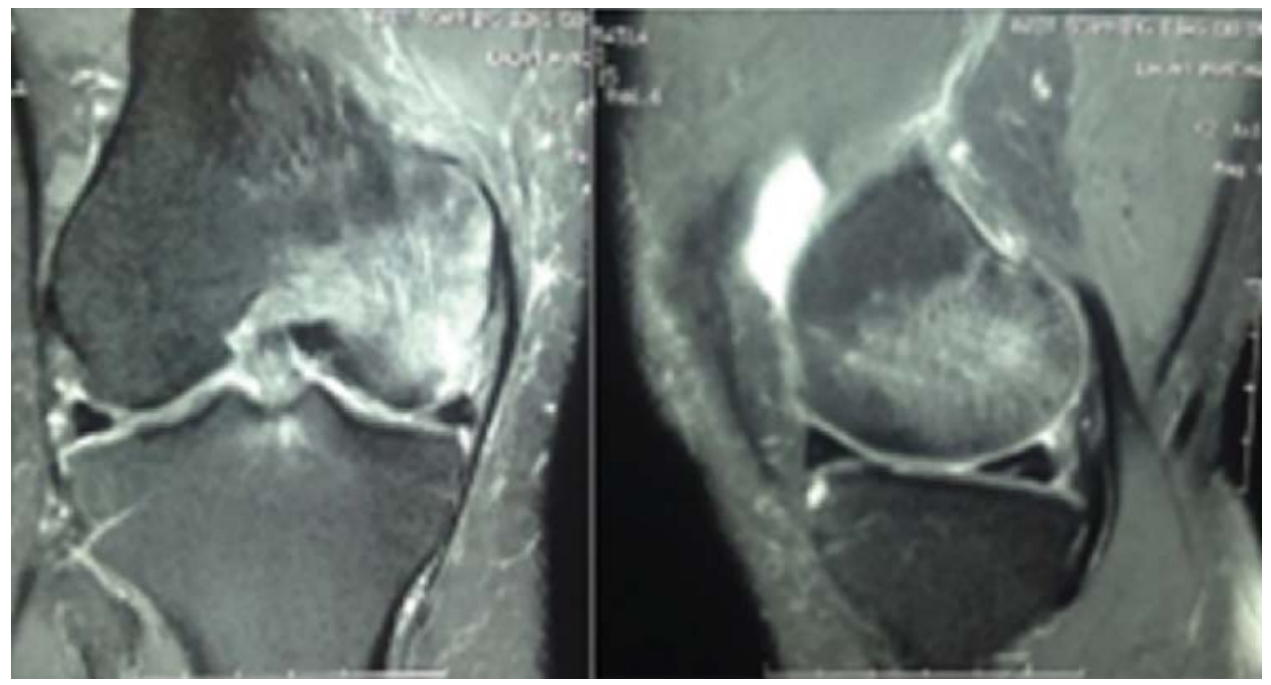

Fig. 1 Cortes coronais e sagitais da articulação do joelho em imagem de RM ponderada em T2 do pré-tratamento mostrando edema ósseo envolvendo mais da metade do côndilo femoral medial.
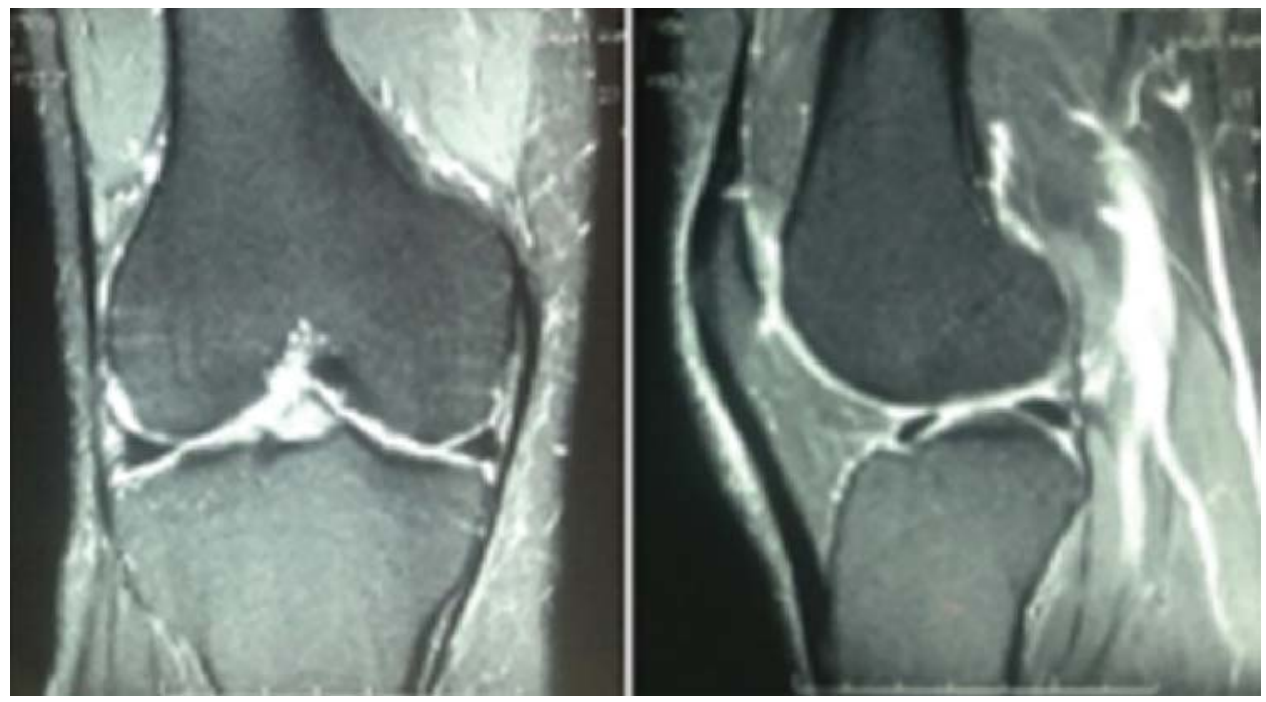

Fig. 2 Cortes coronais e sagitais da articulação do joelho em imagem de RM ponderada em T2 do pós-tratamento mostrando a quase completa reabsorção do edema ósseo após 16 semanas de terapia combinada. 


\section{Resultados}

Dados de 11 pacientes ( 6 mulheres e 5 homens) com ONEJ femoral confirmada por RM foram analisados entre março de 2015 e julho de 2016. A média de idade dos pacientes foi de 52 anos (variação de 35 a 62 anos). Todos os pacientes tinham acometimento articular unilateral (6 do lado direito), com 10 envolvendo o côndilo femoral medial, enquanto 1 tinha um côndilo femoral lateral com ONEJ. Não se incluiu ONEJ o côndilo tibial no estudo. A média da pontuação da EVA no início da terapia foi de 7,72, e de 0,81 com 16 semanas de terapia, sendo a diferença estatisticamente significativa $(p=0,03)$. Em geral, a dor diminuiu consideravelmente após 3 semanas do início da terapia, como foi descrito pelos pacientes, mas a pontuação da EVA não foi calculada neste momento.
A dosagem e a frequência de necessidade de analgésicos de todos os pacientes diminuíram ao longo do tempo, e a duração máxima da ingestão analgésica registrada foi de 8 semanas, após as quais não foi necessário o uso de analgésicos. A média da pontuação da EVA em 8 semanas foi de 1,64, a diferença neste momento também sendo estatisticamente significativa $(p \leq 0,05)$. Até 16 semanas nenhum paciente pediu para suspender o uso de alendronato oral devido a qualquer efeito colateral importante. $O$ envolvimento médio da medula óssea em 0 semanas era de $80 \%$, sendo significativamente reduzido para uma média de $11,81 \%$ em 16 semanas $(p=0,03)$ (-Figuras 1-7). Três pacientes tinham cistos subcondrais de pequeno volume, todos com menos de $14 \mathrm{~mm}$ de comprimento e $4 \mathrm{~mm}$ de largura, e todos apresentaram alta
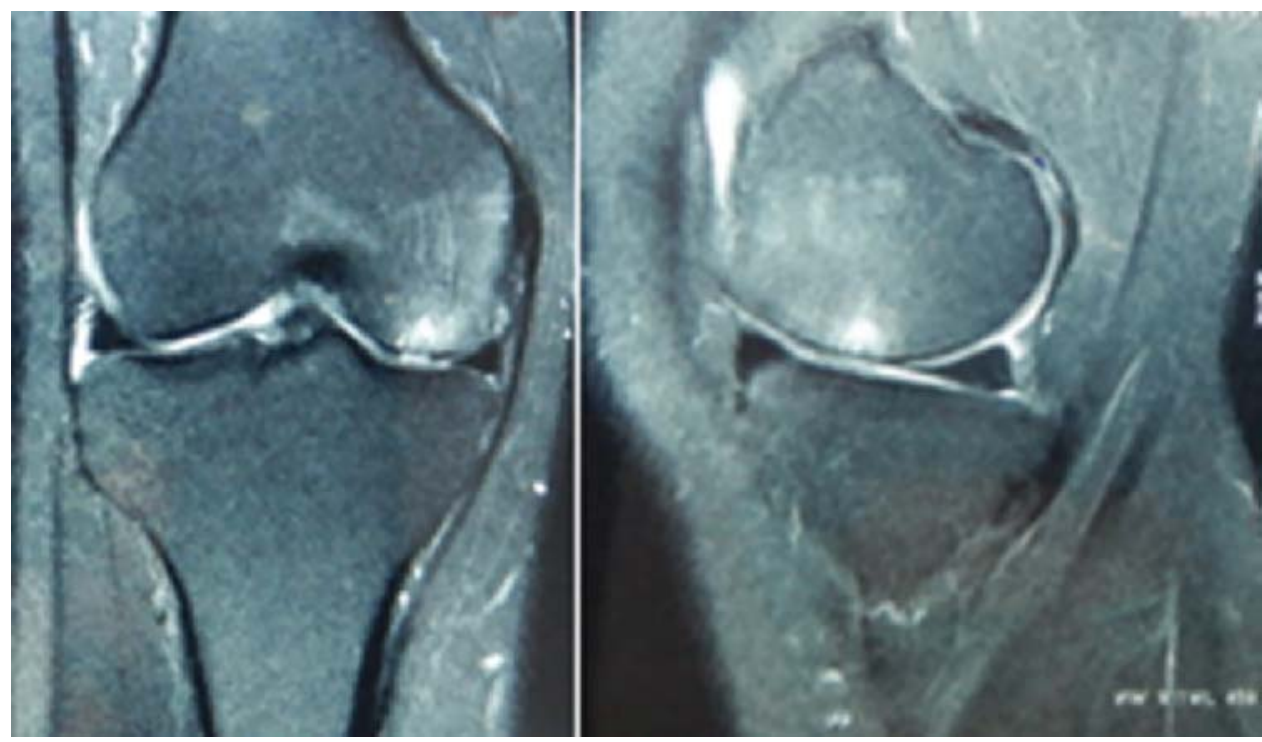

Fig. 3 Cortes coronais e sagitais da articulação do joelho em imagem de RM ponderada em T2 do pré-tratamento mostrando edema ósseo envolvendo $70 \%$ do côndilo femoral medial juntamente com cisto subcondral, afinamento da cartilagem articular, e alterações de contorno da cartilagem.

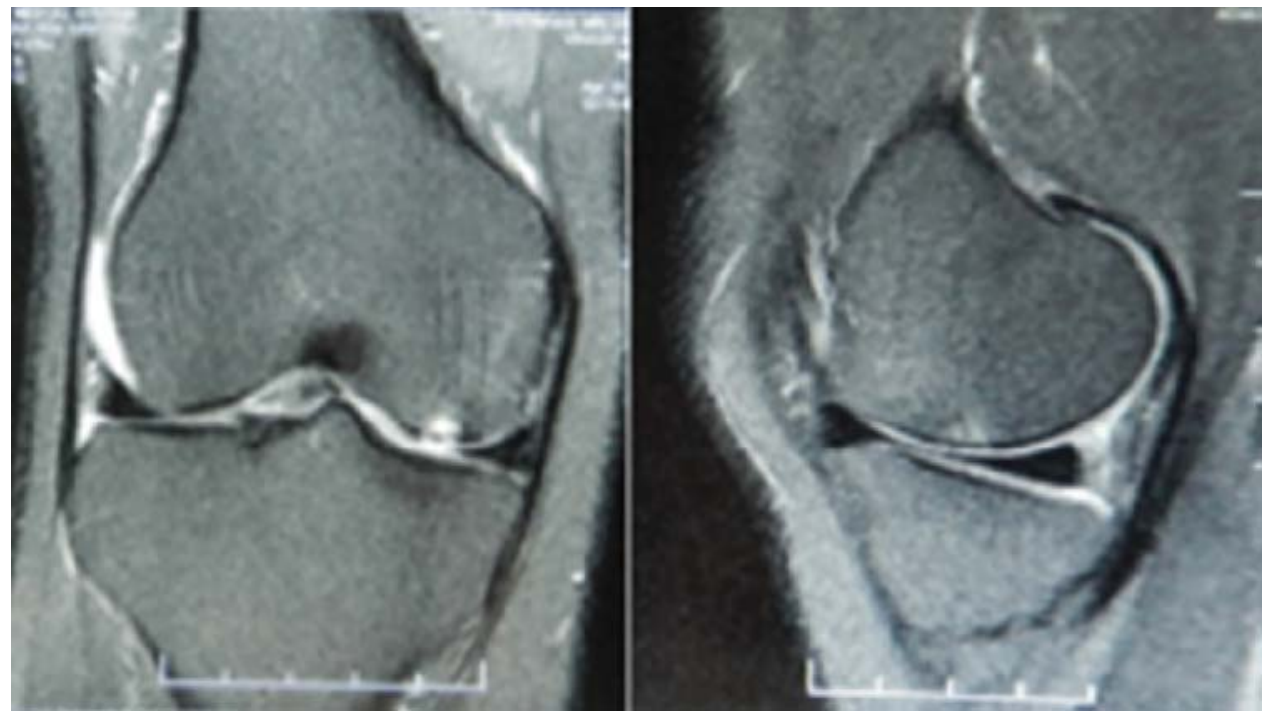

Fig. 4 Cortes coronais e sagitais da articulação do joelho em imagem de RM ponderada em T2 do pós-tratamento mostrando completa reabsorção do edema ósseo após 16 semanas de terapia combinada. 


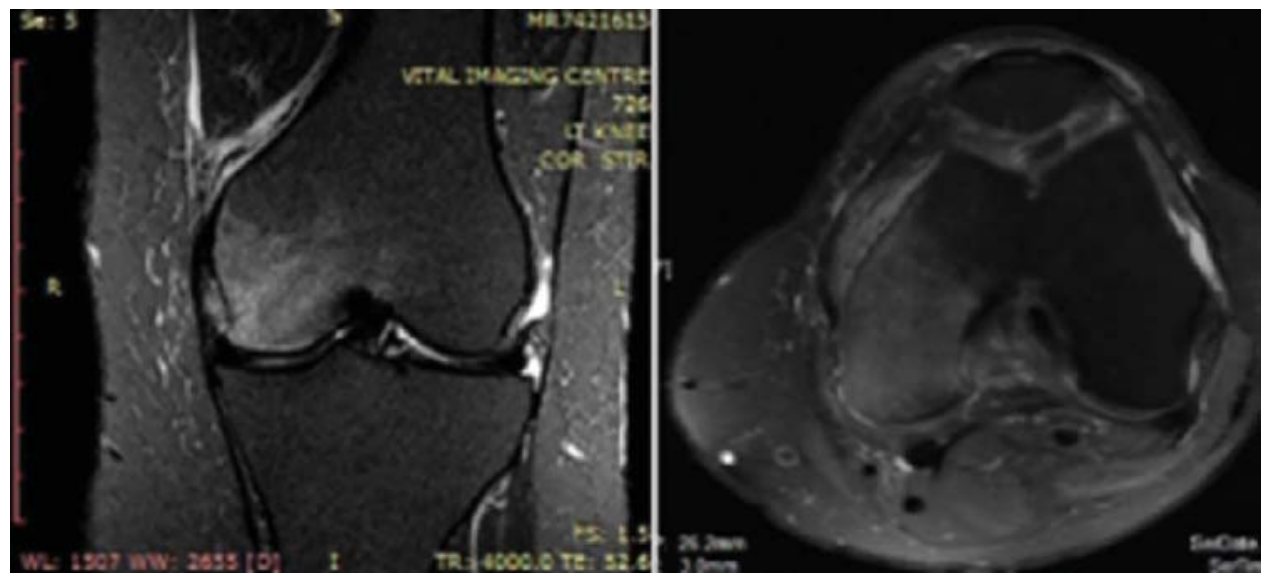

Fig. 5 Cortes coronais e sagitais da articulação do joelho em imagem de RM ponderada em T2 do pré-tratamento mostrando edema ósseo envolvendo cerca de $90 \%$ do côndilo femoral medial.

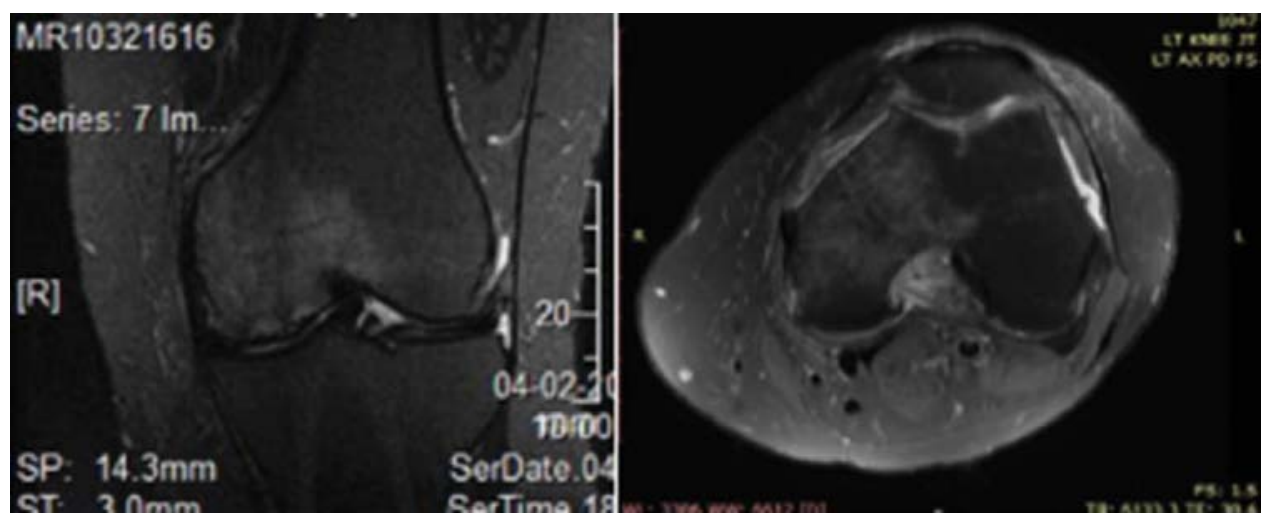

Fig. 6 Cortes coronais e sagitais da articulação do joelho em imagem de RM ponderada em T2 do pós-tratamento mostrando reabsorção considerável do edema ósseo após 16 semanas de terapia combinada.

\section{Porcentagem média pré e pós-tratamento do côndilo envolvido com o edema ósseo}

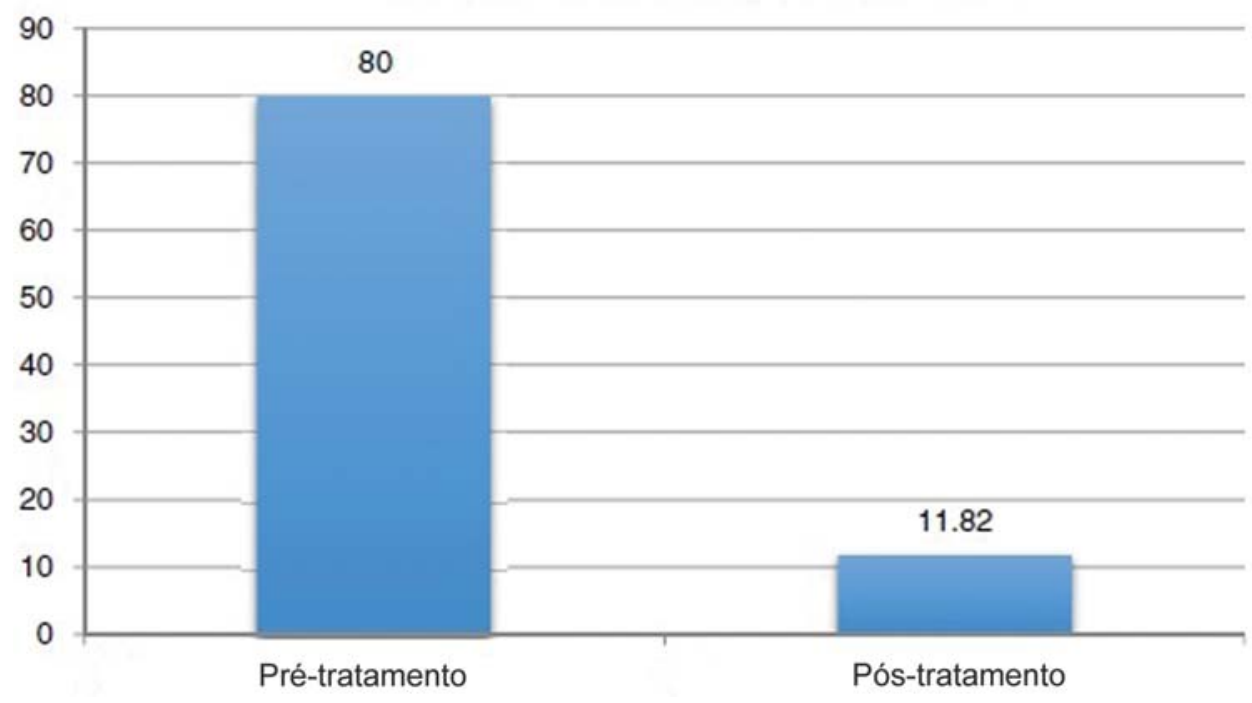

Fig. 7 Diagrama de barras mostrando a porcentagem de edema ósseo envolvendo um único côndilo do fêmur no pré e no pós-tratamento. 1 Porcentagem média pré e pós-tratamento do côndilo envolvido com o edema ósseo 2-Pré-tratamento 3-Pós-tratamento 
intensidade de sinal nas imagens ponderadas em $\mathrm{T} 2$, que foram critérios para um bom prognóstico de acordo com Leucovet et al. ${ }^{19}$ Um total de 5 pacientes tiveram osteoartrite $(\mathrm{OA})$ concomitante, e 3 pacientes apresentaram lesões na raiz do menisco; nenhum paciente teve alterações do contorno articular. Nenhuma conclusão definitiva poderia ser feita para os possíveis fatores de risco de ONEJ devido ao pequeno tamanho da amostra.

\section{Discussão}

Em nosso estudo, descobrimos que a combinação de AZ com alendronato leva a uma redução mais rápida e significativa do EMO. Houve uma redução significativa na pontuação da EVA após 8 e 16 semanas do início da terapia. Todos os pacientes começaram a a suportar seu peso total após 16 semanas de tratamento, sem dor, e descreveram o tratamento como muito eficaz sintomaticamente. A amplitude de movimento da articulação do joelho estava livre de dor às 16 semanas para todos os pacientes. Lecouvet et al. ${ }^{19}$ estudaram as características das imagens de RM para diferenciar e prognosticar osteonecrose irreversível e lesões transitórias do côndilo femoral com EMO. Segundo esse estudo, áreas subcondrais grossas de baixa intensidade de sinal em imagens ponderadas em T2, linhas finas de baixa intensidade de sinal localizadas profundamente no osso trabecular, e deformidades focais sutis do contorno epifisário são as características mais importantes associadas a um estágio inicial de osteonecrose irreversível. A maioria dos pacientes do nosso estudo apresentou "bom prognóstico" de acordo com os critérios descritos na literatura por Lecouvet et al. ${ }^{19}$ Atualmente, não há terapia farmacológica padrão disponível para ONEJ.

Laslett et al. ${ }^{20}$ realizaram um estudo para comparar o efeito de uma única infusão de $A Z$ com placebo em pacientes com OA clínica do joelho. Após seis meses de terapia, eles descobriram que a redução na lesão total da medula óssea foi significativamente maior no grupo $A Z$ do que no grupo placebo. ${ }^{20}$ Essa evidência formou a base de nossa estratégia para incorporar o AZ como parte de nossa terapia combinada.

A biodisponibilidade oral do alendronato no estômago vazio é de apenas $0,7 \%$, e o alimento inibe acentuadamente a absorção por via oral. A farmacocinética do alendronato é estudada exclusivamente com base na sua excreção urinária, uma vez que concentrações plasmáticas extremamente baixas são alcançadas após a ingestão. ${ }^{21} \mathrm{O}$ início lento da ação do alendronato começa apenas após um mês de ingestão da substância. ${ }^{22}$ A administração intravenosa de AZ tem 100\% de biodisponibilidade, e o início de ação é mais rápido do que a do alendronato. ${ }^{23}$ Saag et al. ${ }^{24}$ relataram que níveis mais baixos de telopeptídeo N terminal (NTX) urinário após a administração de $5 \mathrm{mg}$ de AZ foram obtidos após uma semana de início da terapia, enquanto que com $70 \mathrm{mg}$ de alendronato semanalmente por grupo isso demora 12 semanas. Esses dados mostram que a combinação de $\mathrm{AZ}$ com alendronato garante o início precoce de ação e manutenção sustentada. Também acreditamos que o tratamento de uma condição dolorosa aguda como a ONEJ requer tratamento inicial agressivo, usando uma modalidade de ação mais rápida seguida de administração sustentada de bisfosfonatos, ao contrário de uma condição como a osteoporose, que requer bisfosfonatos de baixa dosagem e longa ação.

Altas doses de AZ são administradas em condições como complicações relatadas por metástases. Um estudo de autoria de Berenson, ${ }^{25}$ de ensaios clínicos de fase 3 , em mais de 3 mil pacientes que receberam $4 \mathrm{mg}$ de $\mathrm{AZ}$ por via intravenosa a cada 3 a 40 semanas para metástase em condições como mieloma múltiplo, câncer de mama, câncer de próstata ou outros tumores sólidos, demonstrou que essa droga é segura e eficaz. ${ }^{25}$ Assim, geralmente, cerca de 12 a $16 \mathrm{mg}$ de $\mathrm{AZ}$ são administrados em um curto período de 9 a 16 semanas. Esse estudo mostra que altas doses de bisfosfonatos são justificadas para uma patologia óssea dolorosa e aguda. Embora a analogia elaborada seja indireta, o autor baseou o raciocínio do estudo em uma sólida compreensão da condição, na experiência clínica no manejo da ONEJ, e na farmacocinética e farmacodinâmica dos bisfosfonatos.

Jureus et al. ${ }^{16}$ realizaram um estudo prospectivo com 17 pacientes com lesão de ONEJ que foram tratados com alendronato, administrando semanalmente $70 \mathrm{mg}$ via oral por um período mínimo de 6 meses (média de 11 meses), e os pacientes foram acompanhados clínica e radiograficamente por 1 ano de terapia. Desses, 59\% tiveram recuperação radiográfica completa, contra $25 \%$ dos casos não tratados do mesmo hospital. Estes resultados são semelhantes aos do nosso estudo. Meier et al. ${ }^{17}$ fizeram um estudo controlado e randomizado da eficácia do ibandronato em ONEJ comparado a um placebo. Um total de 30 pacientes foram randomizados para receber o ibandronato intravenoso ou o placebo por 12 semanas, e foram acompanhados por 48 semanas em termos de escore de dor, mobilidade e resultado radiológico. Eles relataram que os bisfosfonatos não trouxeram benefícios adicionais além daqueles dos medicamentos anti-inflamatórios em 12 semanas ou 48 semanas, tanto funcional quanto radiograficamente. Nosso estudo difere desse, pois usamos uma combinação de bisfosfonatos. Raynauld et al. ${ }^{26}$ analisaram durante 24 meses o impacto do risedronato na medula óssea e em lesões císticas do joelho por RM. O estudo teve 4 grupos de tratamento: placebo, $5 \mathrm{mg}$ de risedronato por dia, $15 \mathrm{mg}$ de risedronato por dia, e $50 \mathrm{mg}$ de risedronato por dia, durante 24 meses. Os autores não descreveram nenhuma vantagem dos bisfosfonatos com relação ao tratamento com placebo em cistos e lesões da medula óssea na RM.

Kraenzlin et al. $^{3}$ avaliaram 28 pacientes com osteonecrose do joelho tratados com combinação de pamidronato e terapia com alendronato (inicialmente, $120 \mathrm{mg}$ de pamidronato intravenoso divididos em 3 a 4 perfusões durante 2 semanas, seguidos por $70 \mathrm{mg}$ de alendronato por via oral semanalmente durante 4 a 6 meses). Houve redução de $80 \%$ na pontuação da EVA 6 meses após o tratamento. ${ }^{3,10}$ Os resultados desse estudo são semelhantes aos nossos - redução de $89 \%$ no escore da EVA $(7,72-0,81)-$, mas a diferença em nosso estudo foi que nossos resultados foram avaliados em 16 semanas (4 meses) de terapia, o que mostra que a resolução dos sintomas foi muito mais rápida. Pamidronato combinado com alendronato foi bem tolerado pelos pacientes. Reações agudas devido ao pamidronato intravenoso foram observadas em 4 pacientes, que não tiveram outros efeitos colaterais devido ao 
alendronato oral. Todos os pacientes completaram o curso pretendido de terapia sem interrupção.

O mecanismo da ONEJ parece similar ao da necrose avascular da cabeça femoral. Nishii et al. realizaram um estudo para monitorar a progressão do colapso da cabeça femoral em pacientes com osteonecrose tratados com terapia oral de alendronato. Esse estudo mostrou uma menor taxa de colapso da cabeça femoral, e os autores relataram menor dor no quadril no grupo da necrose quando comoparado com o grupo de controle. ${ }^{27}$

Bisfosfonatos contendo nitrogênio (alendronato, risedronato, ibandronato, pamidronato e AZ) inibem seletivamente a farnesil pirofosfato sintase (FPFS) dentro dos osteoclastos. ${ }^{28}$ Os bisfosfonatos diminuem os parâmetros de reabsorção, como a extensão da área ativamente reabsorvente e a profundidade da erosão medida a partir da superfície óssea. ${ }^{29}$ Estes efeitos detém a reabsorção óssea progressiva e o colapso do osso. A administração de AZ é conveniente, pois requer uma única dose intravenosa por ano e, portanto, a adesão ao tratamento não é um problema.

\section{Limitações do Estudo}

Este foi um estudo retrospectivo com uma pequena amostra e nenhum grupo de controle. A correlação entre a ONEJ e progressão da OA não pôde ser estabelecida, pois se tratou de um estudo de curto prazo. Os fatores de risco da ONEJ também não puderam ser estabelecidos devido ao pequeno tamanho da amostra.

\section{Pontos Fortes do Estudo}

Apesar das limitações, esses resultados promissores preliminares do presente estudo, tanto de avaliações objetivas quanto subjetivas, forneceram resultados consistentes e mostraram que o $\mathrm{AZ}$ combinado com o alendronato tem um efeito de composição, e proporciona melhora sintomática e radiológica em 4 meses, que é mais rápida em comparação com outras modalidades relatadas na literatura. Pelo que sabemos, este é o primeiro estudo que avalia a terapia combinada de $\mathrm{AZ}$ e alendronato para a ONEJ.

\section{Conclusão}

Este estudo de validação de conceito confirma que uma única injeção de $\mathrm{AZ}$ combinada com alendronato para a ONEJ proporciona recuperação mais rápida tanto clínica quanto radiograficamente, em comparação com AZ e/ou ibandronato isoladamente, como descrito na literatura. Estudos posteriores, preferencialmente cegos, ensaios controlados randomizados e com tamanho de amostra maior, são necessários para reafirmar a eficácia do $\mathrm{AZ}$ combinado com o alendronato com relação a outros bisfosfonatos usados isoladamente para ONEJ.

\section{Mensagens-chave}

1. Terapia combinada de bisfosfonatos pode ser administrada com segurança para uma entidade patológica dolorosa como a ONEJ.
2. A história natural da ONEJ pode ser bastante reduzida com o nosso tratamento.

3. A melhora sintomática é confirmada por resultados radiológicos que podem evitar a necessidade de cirurgia.

\section{Conflito de Interesses}

Os autores declaram não haver conflito de interesses.

Agradecimentos

Os autores agradecem aos Dr. V.R. Joshi e Dr. Pratishtha Banga pelo apoio na escrita do artigo.

\section{Referências}

1 Ahlbäck S, Bauer GC, Bohne WH. Spontaneous osteonecrosis of the knee. Arthritis Rheum 1968;11(06):705-733

2 Juréus J, Lindstrand A, Geijer M, Robertsson O, Tägil M. The natural course of spontaneous osteonecrosis of the knee (SPONK): a 1- to 27-year follow-up of 40 patients. Acta Orthop 2013;84(04): 410-414

3 Kraenzlin ME, Graf C, Meier C, Kraenzlin C, Friedrich NF. Possible beneficial effect of bisphosphonates in osteonecrosis of the knee. Knee Surg Sports Traumatol Arthrosc 2010;18 (12):1638-1644

4 Yamamoto T, Bullough PG. Spontaneous osteonecrosis of the knee: the result of subchondral insufficiency fracture. J Bone Joint Surg Am 2000;82(06):858-866

5 Choy WS, Kim KJ, Lee SK, Yang DS, Kim CM, Park JS. Medial unicompartmental knee arthroplasty in patients with spontaneous osteonecrosis of the knee. Clin Orthop Surg 2011;3(04):279-284

6 Mears SC, McCarthy EF, Jones LC, Hungerford DS, Mont MA. Characterization and pathological characteristics of spontaneous osteonecrosis of the knee. Iowa Orthop J 2009;29:38-42

7 Aglietti P, Insall JN, Buzzi R, Deschamps G. Idiopathic osteonecrosis of the knee. Aetiology, prognosis and treatment. J Bone Joint Surg Br 1983;65(05):588-597

8 Son IJ, Kim MK, Kim JY, Kim JG. Osteonecrosis of the knee after arthroscopic partial meniscectomy. Knee Surg Relat Res 2013;25 (03):150-154

9 Houpt JB, Pritzker KP, Alpert B, Greyson ND, Gross AE. Natural history of spontaneous osteonecrosis of the knee (SONK): a review. Semin Arthritis Rheum 1983;13(02):212-227

10 Karim AR, Cherian JJ, Jauregui JJ, Pierce T, Mont MA. Osteonecrosis of the knee: review. Ann Transl Med 2015;3(01):6

11 Reddy AS, Frederick RW. Evaluation of the intraosseous and extraosseous blood supply to the distal femoral condyles. Am J Sports Med 1998;26(03):415-419

12 Satku K, Kumar VP, Chong SM, Thambyah A. The natural history of spontaneous osteonecrosis of the medial tibial plateau. J Bone Joint Surg Br 2003;85(07):983-988

13 Lotke PA, Abend JA, Ecker ML. The treatment of osteonecrosis of the medial femoral condyle. Clin Orthop Relat Res 1982;(171): 109-116

14 Agarwala S, Sule A, Pai BU, Joshi VR. Alendronate in the treatment of avascular necrosis of the hip. Rheumatology (Oxford) 2002;41 (03):346-347

15 Agarwala S, Shah S, Joshi VR. The use of alendronate in the treatment of avascular necrosis of the femoral head: follow-up to eight years. J Bone Joint Surg Br 2009;91(08):1013-1018

16 Jureus J, Lindstrand A, Geijer M, Roberts D, Tägil M. Treatment of spontaneous osteonecrosis of the knee (SPONK) by a bisphosphonate. Acta Orthop 2012;83(05):511-514

17 Meier C, Kraenzlin C, Friederich NF, et al. Effect of ibandronate on spontaneous osteonecrosis of the knee: a randomized, doubleblind, placebo-controlled trial. Osteoporos Int 2014;25(01): 359-366 
18 Jäger M, Tillmann FP, Thornhill TS, et al. Rationale for prostaglandin $\mathrm{I} 2$ in bone marrow oedema-from theory to application. Arthritis Res Ther 2008;10(05):R120

19 Lecouvet FE, van de Berg BC, Maldague BE, et al. Early irreversible osteonecrosis versus transient lesions of the femoral condyles: prognostic value of subchondral bone and marrow changes on MR imaging. AJR Am J Roentgenol 1998;170(01):71-77

20 Laslett LL, Doré DA, Quinn SJ, et al. Zoledronic acid reduces knee pain and bone marrow lesions over 1 year: a randomised controlled trial. Ann Rheum Dis 2012;71(08):1322-1328

21 Porras AG, Holland SD, Gertz BJ. Pharmacokinetics of alendronate. Clin Pharmacokinet 1999;36(05):315-328

22 Vallerand AH, Deglin JH, Sanoski CA. Davis's drug guide for nurses. 14th ed. Philadelphia: F.A. Davis Company; 2014

23 Lewiecki EM. Intravenous zoledronic acid for the treatment of osteoporosis: The evidence of its therapeutic effect. Core Evid 2010;4:13-23

24 Saag K, Lindsay R, Kriegman A, Beamer E, Zhou W. A single zoledronic acid infusion reduces bone resorption markers more rapidly than weekly oral alendronate in postmenopausal women with low bone mineral density. Bone 2007;40(05): 1238-1243

25 Berenson JR. Recommendations for zoledronic acid treatment of patients with bone metastases. Oncologist 2005;10(01): $52-62$

26 Raynauld JP, Martel-Pelletier J, Berthiaume MJ, et al. Correlation between bone lesion changes and cartilage volume loss in patients with osteoarthritis of the knee as assessed by quantitative magnetic resonance imaging over a 24 -month period. Ann Rheum Dis 2008;67(05):683-688

27 Nishii T, Sugano N, Miki H, Hashimoto J, Yoshikawa H. Does alendronate prevent collapse in osteonecrosis of the femoral head? Clin Orthop Relat Res 2006;443(443):273-279

28 Drake MT, Clarke BL, Khosla S. Bisphosphonates: mechanism of action and role in clinical practice. Mayo Clin Proc 2008;83(09): 1032-1045

29 Rodan GA, Fleisch HA. Bisphosphonates: mechanisms of action. J Clin Invest 1996;97(12):2692-2696 